\title{
Mutually Exclusive Formation of G-quadruplex and i-Motif is a General Phenomenon Governed by Steric Hindrance in Duplex DNA
}

\author{
Yunxi Cui ${ }^{\mathrm{a}}$, Deming Kong ${ }^{\mathrm{b}, *}$, Chiran Ghimire ${ }^{\mathrm{a}}$, Cuixia Xu ${ }^{\mathrm{a}, \mathrm{c}}$, and Hanbin Mao ${ }^{\mathrm{a},{ }^{*}}$ \\ ${ }^{a}$ Department of Chemistry and Biochemistry and School of Biomedical Sciences, Kent State University, Kent, \\ Ohio 44242, United States \\ ${ }^{\mathrm{b}}$ Collaborative Innovation Center of Chemical Science and Engineering (Tianjin), Tianjin, 300071, P R China \\ ${ }^{c}$ MOE Key Laboratory of Bioinorganic and Synthetic Chemistry, School of Chemistry and Chemical \\ Engineering, Sun Yat-Sen University, Guangzhou 510275, China \\ * To whom correspondence should be addressed. Hanbin Mao: Tel: +1 330672 9380, Fax: +1 \\ 330672 3816, Email: hmao@kent.edu. Deming Kong: Tel: +86-22-23500938, Email: \\ kongdem@nankai.edu.cn.
}




\section{Content}

\section{Paragraph}

1.1 Preparation of DNA construct that contains single-stranded G- or C-rich sequences

1.2 Calculation of the change in unfolding free energy of the tetraplex structures

1.3 Calculation of the change-in-the-contour-length $(\Delta L)$ for the unfolding events in DNA fragments

\section{Figures}

2.1. The additivity of the unfolding force

2.2. Population analysis of double-stranded ILPR structure

2.3. Typical F-x curves observed in the analysis of telomere sequence

2.4. Force and $\Delta \mathrm{L}$ histogram obtained in the BCL-2 analysis

2.5. Typical F-x curves observed in the analysis of BCL-2 promoter sequence

2.6. Different populations obtained in the analysis of BCL-2 promoter sequence

\section{Tables}

3.1. DNA sequence applied in the mismatch design

3.2. Percentage of simultaneous formation of the two tetraplex structures

3.3. Possible tetraplex structures of particular sizes in BCL-2 promoter sequence

3.4. Single-stranded wild-type tetraplex forming sequences used in the manuscript. 
Preparation of DNA construct that contains single-stranded G- or C-rich sequences

The single-stranded DNA constructs (human telomeres and BCL-2 promoter, see Table S4 for sequences) used for mechanical unfolding and refolding experiments were prepared by sandwiching the single-stranded target sequences between two double-stranded DNA handles according to the procedures described previously.(1) The 2690-bp and 2028-bp double-stranded DNA handles were prepared as described in the Material and Methods of the manuscript. An ssDNA fragment that contained a target sequence (Table S4) was hybridized with two short single-stranded DNA oligos (5'-CGC ATC TGT GCG GTA TTT CAC ACC GT-3' and 5'-GGC CGA CGC GCT GGG CTA CGT CTT GCT GGC-3'), forming a dsDNA-ssDNA-dsDNA hybrid with EagI and XbaI overhangs at the end of each dsDNA component in the construct. This hybrid was then ligated to the 2690-bp (ended with an EagI overhang) and 2028-bp (ended with an XbaI overhang) dsDNA handles by sequential ligation using T4 DNA ligase.

\section{Calculation of the change in unfolding free energy of the tetraplex structures}

We calculated the change in the free energy of unfolding DNA tetraplex structures ( $\left.\Delta G_{\text {unfold }}\right)$ using the Jarzynski equality (2) (Equation S1):

$$
\Delta G_{\mathrm{unfold}}=-k_{\mathrm{B}} T \ln \sum_{\mathrm{i}=1}^{N} \frac{1}{N} \exp \left(-\frac{w_{\mathrm{i}}}{k_{\mathrm{B}} T}\right)
$$

Where $N$ is the number of repetitions, $w_{\mathrm{i}}$ is the non-equilibrium work done to unfold the structure, $k_{B}$ is the Boltzmann constant, and $T$ is absolute temperature.

\section{Calculation of the change-in-the-contour-length $(\Delta L)$ for the unfolding events in DNA fragments}

The Force-eXtension (F-X) curves recorded from different DNA constructs were filtered by Savitzky-Golay function with a $10 \mathrm{~ms}$ time constant in Matlab program (The Math Works, Nattick, MA). The change in extension $(\Delta x)$ at a given force was calculated from the difference of the extension between stretching and relaxing curves at that force. The change in contour length $(\Delta L)$ was calculated based on the $\Delta x$ by the extensible worm like chain model (WLC) $(3,4)$ (Equation S2),

$$
\frac{\Delta x}{\Delta L}=1-\frac{1}{2} \sqrt{\frac{k_{B} T}{F P}}+\frac{F}{S}
$$

here, $L$ is the contour length, $P$ is the persistence length $(50.8 \mathrm{~nm}), S$ is the stretching modulus (1243 pN) for double-stranded DNA handles.(5)

The expected $\Delta L$ from a folded structure was calculated by Equation $\mathrm{S} 3,(6)$

$$
\Delta L=L_{\mathrm{nt}} * N-x
$$

where $x$ is the end-to-end distance for G-quadruplex or i-motif structures ( 0.5 and $1.5 \mathrm{~nm}$ respectively)(7) and $L_{\mathrm{nt}}$ is the contour length per nucleotide. $L_{\mathrm{nt}}$ is in the range of $0.40-0.45 \mathrm{~nm} / \mathrm{nt}$ for single-stranded $\mathrm{DNA}(8)$ and $0.34 \mathrm{~nm} / \mathrm{bp}$ for 
double-stranded DNA.(9) For a double-stranded DNA with overlapped G-quadruplex and i-motif hosting regions in opposite strands, the number of nucleotides in the folded structure $(N)$ was calculated as,

$$
N=N_{\mathrm{G}-\text { quadruplex }}+N_{\mathrm{i}-\text { motif }}-N_{\text {overlap }} \ldots \ldots \ldots \ldots \ldots \ldots(\mathrm{S} 4)
$$

where $N_{\mathrm{G} \text {-quadruplex }}=N_{\mathrm{i} \text {-motif }}=21 \mathrm{nt}$ for telomeric tetraplexes. The numbers of nucleotides in the overlapped regions $\left(N_{\text {overlap }}\right)$ are $0 \mathrm{nt}$ and $6 \mathrm{nt}$ in the $3 \mathrm{nt}$-out and $6 \mathrm{nt}$-in constructs, respectively. These yield $N=42 \mathrm{nt}$ and $36 \mathrm{nt}$ for the numbers of nucleotides in the double-stranded 3nt-out and 6nt-in constructs, respectively, which correspond to $\Delta L$ of $13.3 \mathrm{~nm}$ and $11.2 \mathrm{~nm}$ using eqn S3, given $1.0 \mathrm{~nm}$ as the end-to-end distance(10) and $0.34 \mathrm{~nm}$ as the contour length per base pair.(11) 


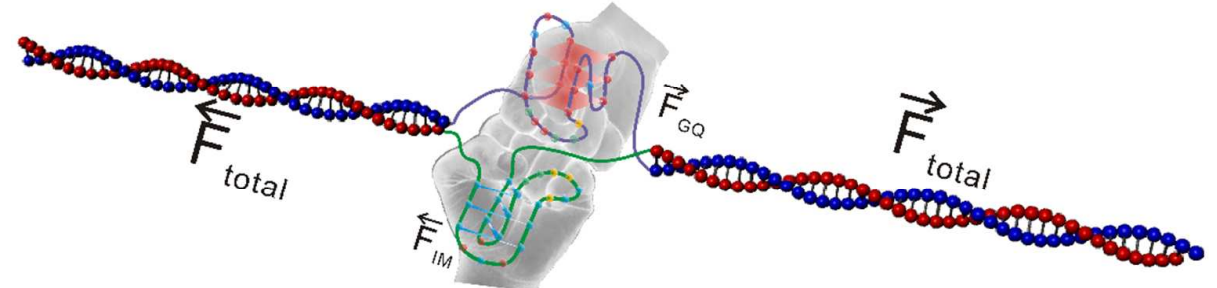

Figure S1: The additivity of the unfolding force of G-quadruplex and i-motif formed simultaneously in the two complementary strands of a duplex DNA (Ftotal=FGQ+FIM). 


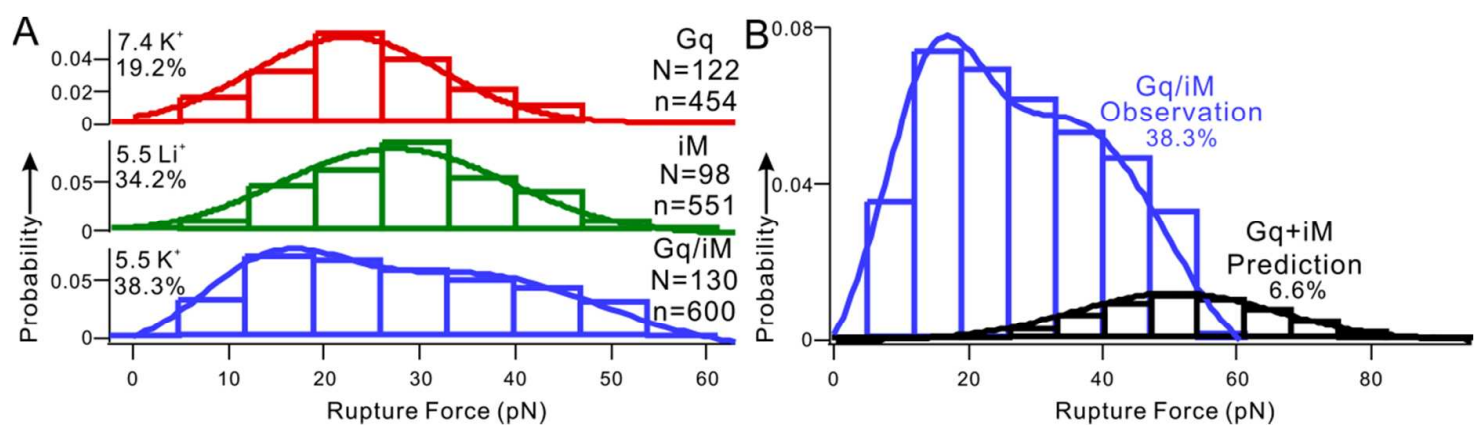

Figure S2: A) Population analyses of ILPR G-quadruplex (Gq) in a $10 \mathrm{mM}$ Tris buffer supplemented with $100 \mathrm{mM} \mathrm{KCl}$ at $\mathrm{pH}$ 7.4 (red), ILPR i-motif (iM) in a $50 \mathrm{mM}$ MES buffer supplemented with $100 \mathrm{mM} \mathrm{LiCl}$ at $\mathrm{pH} 5.5$ (green), and ILPR G-quadruplex and/or i-motif (Gq/iM) in a $50 \mathrm{mM}$ MES buffer supplemented with $100 \mathrm{mM} \mathrm{KCl}$ at pH 5.5 (cyan). B) Predicted probabilities (peak at $\sim 52 \mathrm{pN}$ ) of unfolding both G-quadruplex and i-motif in a $50 \mathrm{mM}$ MES buffer supplemented with 100 $\mathrm{mM} \mathrm{KCl}$ at pH5.5 (black). For comparison, the experimentally observed histogram in this buffer is shown in cyan. Data analyzed here were taken from literature.(12) 

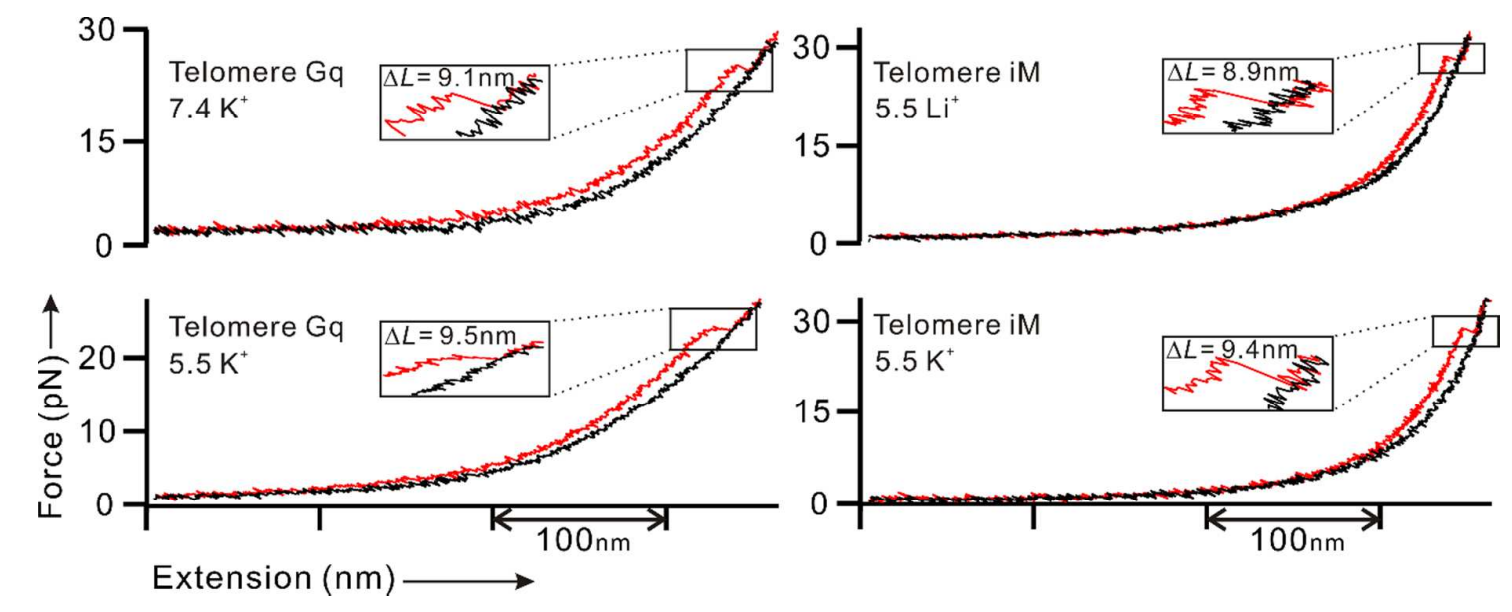

Figure S3. Typical Force-eXtension curves (F-X) of G-quadruplex (Gq, left) and i-motif (iM, right) in single-stranded human telomeric sequences, $5^{\prime}$-TTA(GGGTTA $)_{4}$ and 5'-TAA(CCCTAA $)_{4}$, respectively, in different buffers. See supporting text for the preparation of DNA constructs. Red and black traces represent extending and returning F-X curves respectively. Left panels show telomeric G-quadruplex in a $10 \mathrm{mM}$ Tris buffer supplemented with $100 \mathrm{mM} \mathrm{KCl}$ at pH 7.4 (top) and $50 \mathrm{mM} \mathrm{MES}$ buffer supplemented with $100 \mathrm{mM} \mathrm{KCl}$ at pH 5.5 (bottom). Right panels show telomeric i-motif at pH 5.5 in a $10 \mathrm{mM}$ Tris buffer with $100 \mathrm{mM} \mathrm{LiCl}$ (top) and a $50 \mathrm{mM}$ MES buffer with $100 \mathrm{mM} \mathrm{KCl}$ (bottom). 

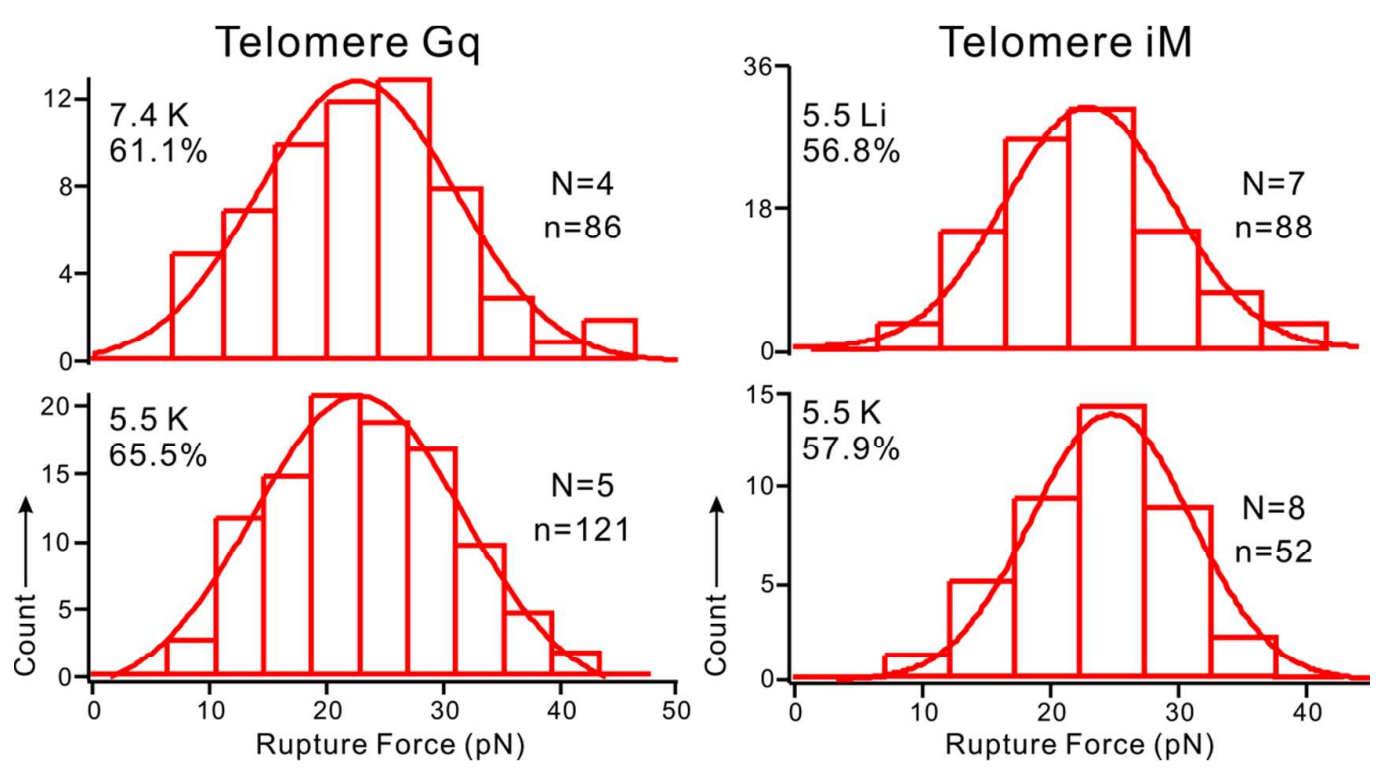

Figure S4: Rupture force histograms of G-quadruplex (Gq, left) and i-motif (iM, right) in single-stranded human telomeric sequences, $5^{\prime}$-TTA $(\text { GGGTTA })_{4}$ and 5'-TAA(CCCTAA $)_{4}$, respectively, in different buffers. Left panels show similar rupture force histograms and percentage formation of telomeric G-quadruplex in a $10 \mathrm{mM}$ Tris buffer supplemented with $100 \mathrm{mM}$ $\mathrm{KCl}$ at $\mathrm{pH} 7.4$ (top, $\mathrm{F}_{\text {rupture }}=22.4 \mathrm{pN} ; 61.1 \%$ formation) and $50 \mathrm{mM}$ MES buffer supplemented with $100 \mathrm{mM} \mathrm{KCl}$ at $\mathrm{pH} 5.5$ (bottom, $\mathrm{F}_{\text {rupture }}=22.7 \mathrm{pN} ; 65.5 \%$ formation). Right panels show similar rupture force histograms and percentage formation of telomeric i-motif at pH 5.5 in a $10 \mathrm{mM}$ Tris buffer with $100 \mathrm{mM} \mathrm{LiCl}$ (top, $\mathrm{F}_{\text {rupture }}=23.5 \mathrm{pN} ; 56.8 \%$ formation) and a $50 \mathrm{mM}$ MES buffer with $100 \mathrm{mM} \mathrm{KCl}$ (bottom, $\mathrm{F}_{\text {rupture }}=24.6 \mathrm{pN} ; 57.9 \%$ formation). $\mathrm{N}$ and $\mathrm{n}$ represent the number of molecules and number of unfolding features, respectively. 

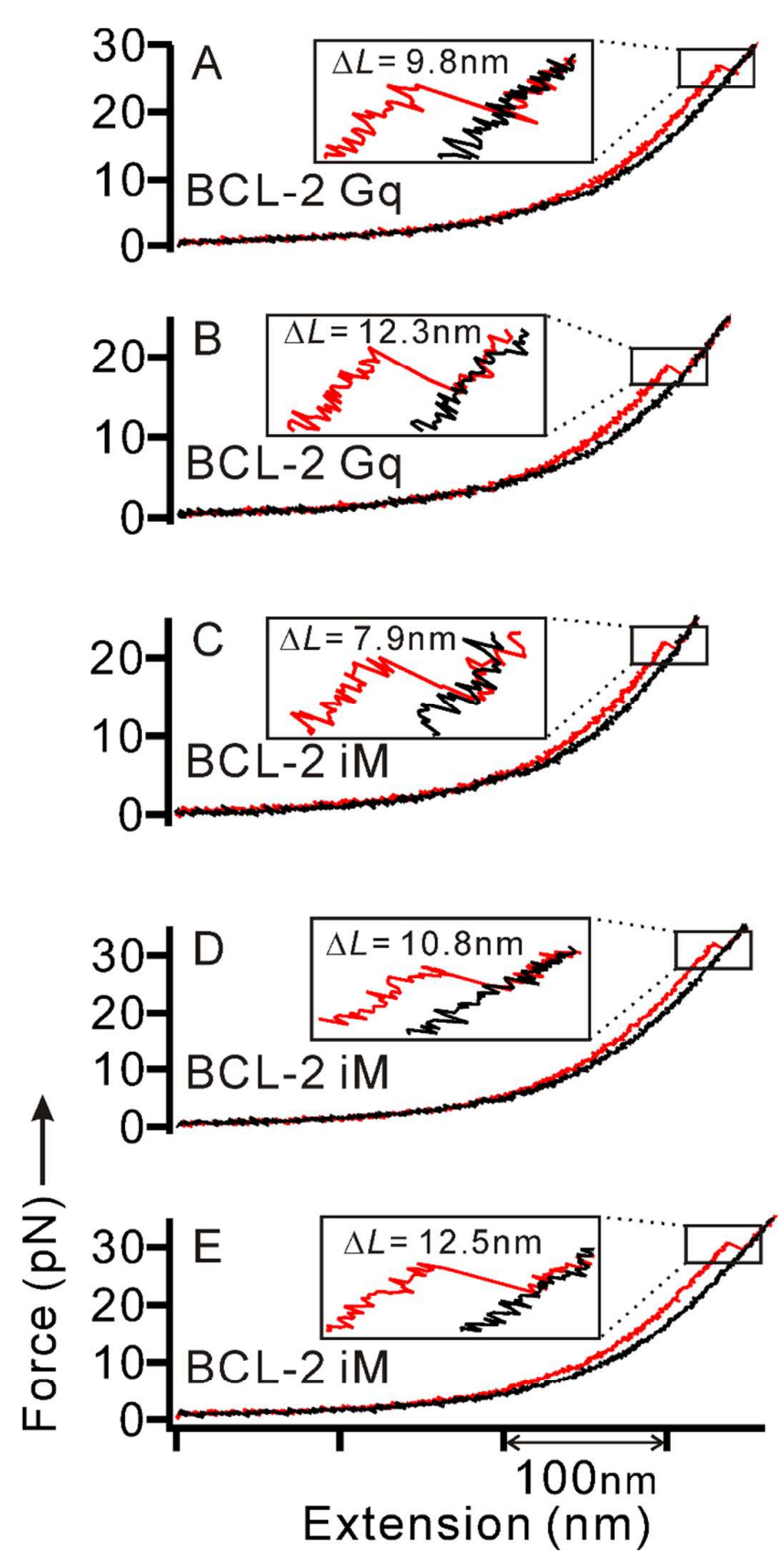

Figure S5. Typical Force-eXtension curves (F-X) of single-stranded G-rich (A and B, 5'-

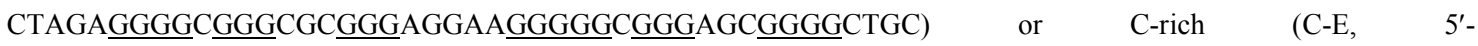
GGCCGCAGCCCCGCTCCCGCCCCCTTCCTCCCGCGCCCGCCCCT) BCL-2 DNA fragments in different buffers. Red and black traces represent extending and returning F-X curves respectively. See supporting text for the preparation of the DNA constructs. Unfolding transitions with different $\Delta \mathrm{L}$ sizes (see insets) were analyzed in Figure S6 statistically. 


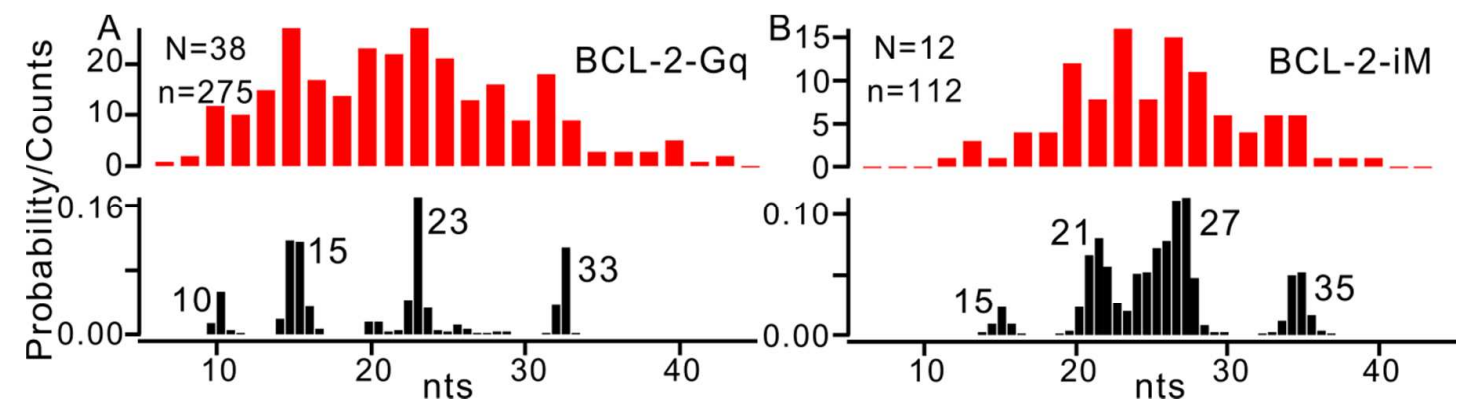

Figure S6. Different tetraplex populations exist in the single-stranded G-rich (A, 5'-

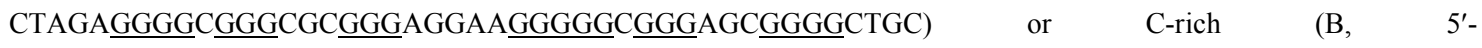
GGCCGCAGCCCCGCTCCCGECCCCTTCCTCCCGCGCCCGCCCCT) BCL-2 promoter sequences, respectively. Red and black histograms depict $\Delta \mathrm{L}$ populations measured at $\mathrm{F}_{\text {rupture }}$ and deconvoluted using the PoDNano method, $(4,13)$ respectively. $\mathrm{N}$ and $\mathrm{n}$ represent the number of molecules and number of unfolding features, respectively. See Table S3 for possible fully-folded tetraplex structures. 
Table S1. Sequence of the DNA used in the offset design in Figure 5. Mutation sites to prevent the formation of either G-quadruplex or i-motif are highlighted.

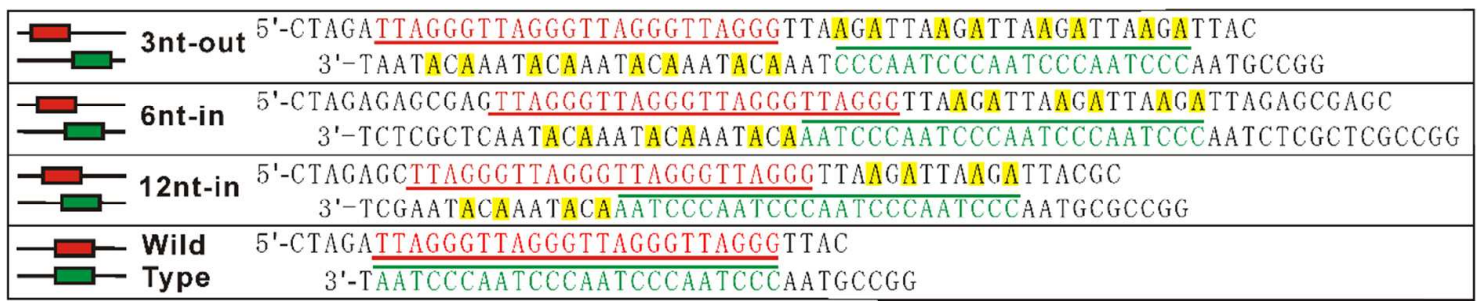


Table S2. Percentage of simultaneous formation of the two tetraplex structures in the telomere DNA constructs shown in Figure 5.

\begin{tabular}{|c|c|c|}
\hline & $\begin{array}{c}\text { Total } \\
\text { tethers }\end{array}$ & Double features (\%) \\
\hline 3nt-out & 217 & $8(3.7 \%)$ \\
\hline 6nt-in & 250 & $4(1.6 \%)$ \\
\hline 12nt-in & 184 & $1(<0.5 \%)$ \\
\hline WT & 468 & $1(<0.2 \%)$ \\
\hline
\end{tabular}


Table S3. Possible tetraplex structures of particular sizes depicted in Figure S6. G/C tracts involved in tetraplex formation are underlined.

\begin{tabular}{|c|c|c|}
\hline \multirow{5}{*}{$\mathrm{Gq}$} & \multirow{4}{*}{$23 \mathrm{nt}$} & $\underline{\text { GGGGCGGGCGCGGGAGGAAGGGG }}$ \\
\hline & & $\underline{\text { GGGCGGGCGCGGGAGGAAGGGGG }}$ \\
\hline & & $\underline{\text { GGGCGCGGGAGGAAGGGGGCGGG }}$ \\
\hline & & $\underline{\text { GGGAGGAAGGGGGCGGGAGCGGG }}$ \\
\hline & $33 \mathrm{nt}$ & $\underline{\text { GGGCGGGCGCGGGAGGAAGGGGGCGGGAGCGGG }}$ \\
\hline \multirow{3}{*}{$\mathrm{iM}$} & $21 \mathrm{nt}$ & 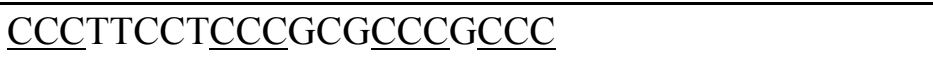 \\
\hline & $27 \mathrm{nt}$ & $\underline{\text { CCCGCCCCCTTCCTCCCGCGCCCGCCC }}$ \\
\hline & $35 \mathrm{nt}$ & СCCCGCTCCCGCCCCCTTCCTCCCGCGCCCGCCCC \\
\hline
\end{tabular}


Table S4. Single-stranded wild-type tetraplex forming sequences (red) used in the manuscript.

Possible G/C tracts for G-quadruplex or i-motifformation are underlined.

\begin{tabular}{|c|c|c|}
\hline \multirow[b]{2}{*}{ Telomere } & G-rich & 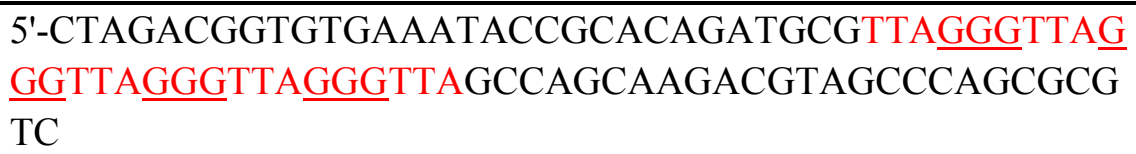 \\
\hline & C-rich & 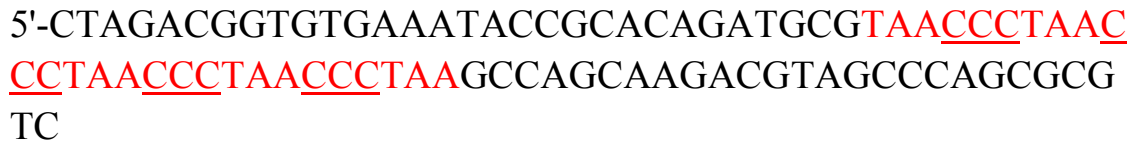 \\
\hline \multirow[b]{2}{*}{ BCL-2 } & G-rich & $\begin{array}{l}\text { 5'-CTAGACGGTGTGAAATACCGCACAGATGCGTTGGGGCGGG } \\
\text { CGCGGGAGGAAGGGGGCGGGAGCGGGGCTGTTGCCAGCAA } \\
\text { GACGTAGCCCAGCGCGTC }\end{array}$ \\
\hline & C-rich & $\begin{array}{l}\text { 5'-CTAGACGGTGTGAAATACCGCACAGATGCGTTCAGCCCCG } \\
\text { CTCCCGCCCCCTTCCTCCCGCGCCCGCCCCTTGCCAGCAAGA } \\
\text { CGTAGCCCAGCGCG TC }\end{array}$ \\
\hline
\end{tabular}




\section{Supporting Reference:}

1. Dhakal, S., Yu, Z., Konik, R., Koirala, D., and Mao, H. (2011) Formation of human ILPR G-quadruplex in dsDNA, International Review of Biophysical Chemistry 2 (Proceeding of 3rd international meeting on G-quadruplexes and G-assembly), 191-193.

2. Jarzynski, C. (1997) Nonequilibrium Equality for Free Energy Differences, Phys. Rev. Lett. 78, 2690 - 2693.

3. Baumann, C. G., Smith, S. B., Bloomfield, V. A., and Bustamante, C. (1997) Ionic effects on the elasticity of single DNA molecules, Proc. Natl. Acad. Sci. USA 94, 6185-6190.

4. Yu, Z., and Mao, H. (2013) Non-B DNA structures show diverse conformations and complex transition kinetics comparable to RNA or proteins - a perspective from mechanical unfolding and refolding experiments, Chem. Rec. 13, 102-116.

5. Dhakal, S., Cui, Y., Koirala, D., Ghimire, C., Kushwaha, S., Yu, Z., Yangyuoru, P. M., and Mao, H. (2013) Structural and mechanical properties of individual human telomeric G-quadruplexes in molecularly crowded solutions, Nucleic Acids Res. 41, 3915-3923.

6. Greenleaf, W. J., Frieda, K. L., Foster, D. A., Woodside, M. T., and Block, S. M. (2008) Direct observation of hierarchical folding in single riboswitch aptamers, Science 319, 630-633.

7. Dhakal, S., Schonhoft, J. D., Koirala, D., Yu, Z., Basu, S., and Mao, H. (2010) Coexistence of an ILPR i-Motif and a Partially Folded Structure with Comparable Mechanical Stability Revealed at the Single-Molecule Level, J. Am. Chem. Soc. 132, 8991-8997.

8. Laurence, T. A., Kong, X., Jager, M., and Weiss, S. (2005) Probing structural heterogeneities and fluctuations of nucleic acids and denatured proteins, Proc. Nat. Acad. Sci. USA 102, 17348-17353.

9. Levitt, M. (1983) How many base-pairs per turn does DNA have in solution and in chromatin? Some theoretical calculations, Proc. Natl. Acad. Sci. U. S. A. 75, 5.

10. Lim, K. W., Lacroix, L., Yue, D. J., Lim, J. K., Lim, J. M., and Phan, A. T. (2010) Coexistence of two distinct G-quadruplex conformations in the hTERT promoter, $J$. Am. Chem. Soc. 132, 12331-12342.

11. Williams, M. C., Wenner, J. R., Rouzina, I., and Bloomfield, V. A. (2001) Entropy and heat capacity of DNA melting from temperature dependence of single molecule stretching, Biophys J 80, 1932-1939.

12. Dhakal, S., Yu, Z., Konik, R., Cui, Y., Koirala, D., and Mao, H. (2012) G-Quadruplex and i-Motif Are Mutually Exclusive in ILPR Double-Stranded DNA, Biophys. J. 102, 2575-2584.

13. Yu, Z., Gaerig, V., Cui, Y., Kang, H., Gokhale, V., Zhao, Y., Hurley, L. H., and Mao, H. (2012) Tertiary DNA Structure in the Single-Stranded hTERT Promoter Fragment 
Unfolds and Refolds by Parallel Pathways via Cooperative or Sequential Events, $J$. Am. Chem. Soc. 134, 5157-5164. 